\title{
Analyses, Simulations and Physical Modeling Validation of Levee and Embankment Erosion
}

Zhongxian Chen ${ }^{1}$; Christopher S. Stuetzle ${ }^{2}$; Barbara M. Cutler ${ }^{3}$; Jared A. Gross ${ }^{4}$, A.M. ASCE; W. Randolph Franklin ${ }^{5}$; and Thomas F. Zimmie ${ }^{6}$, P.E., M.ASCE

${ }^{1}$ Department of Computer Science, Rensselaer Polytechnic Institute, $1108^{\text {th }}$ Street, Troy, NY 12180; email: chenz5@cs.rpi.edu

${ }^{2}$ Department of Computer Science, Rensselaer Polytechnic Institute, $1108^{\text {th }}$ Street, Troy, NY 12180; email: stuetc@cs.rpi.edu

${ }^{3}$ Department of Computer Science, Rensselaer Polytechnic Institute, $1108^{\text {th }}$ Street, Troy, NY 12180; email: cutler@cs.rpi.edu

${ }^{4}$ Department of Civil \& Environmental Engineering, Rensselaer Polytechnic Institute, $1108^{\text {th }}$ Street, Troy, NY 12180; email: grossj3@rpi.edu

${ }^{5}$ Eletronic, Computer, and System Engineering Department, Rensselaer Polytechnic Institute, $1108^{\text {th }}$ Street, Troy, NY 12180; email: frankwr@rpi.edu

${ }^{6}$ Department of Civil \& Environmental Engineering, Rensselaer Polytechnic Institute, $1108^{\text {th }}$ Street, Troy, NY 12180; email: zimmit@rpi.edu

\section{| ABSTRACT}

We present a computer simulation of hydraulic erosion on levees, dams, and earth embankments, with emphasis on rill and gully initiation and propagation. We focus on erosion features that occur after an earthen structure is overtopped. We have developed a 3D fluid and hydraulic erosion simulation engine using Smoothed Particle Hydrodynamics (SPH). We present the results of digital simulations for different soil types. Furthermore, small-scale physical models of levees composed of different soils were constructed and tested experimentally. The digital simulations are compared to physical experimental results to validate the computer models.

\section{INTRODUCTION}

After the devastation of Hurricane Katrina in 2005, much attention has been given to the analysis of erosion and breaching of levees in storms and floods. A primary cause of levee failure is overtopping, although seepage is also a possible cause. A better understanding of how levees are eroded and damaged when overtopped can help engineers design levees that better withstand large storms.

In this paper, we present a digital simulation of hydraulic erosion with a focus on small-scale earthen embankments, specifically the formation of rills and gullies. We have conducted experiments with both our computer simulation and in a physical laboratory. The experiments were carefully designed to match the experimental setup (geometry of the environment, soil parameters, and water flow rate) allowing direct 
comparisons of the results. The digital simulation and real-world experimental results, to date, are presented and compared in this paper.

\section{RELATED WORK}

Simulations of fluid and hydraulic erosion have a long history in the field of Computer Graphics and have received more attention in recent years. This work is primarily concerned with creating physically plausible results (e.g., for movies or video games), and thus little effort has been made to validate the physical accuracy with physical experiments. In the sections below, we provide an introduction to Smoothed Particle Hydrodynamics, applications of SPH in physics and computer science, and existing methods for digital simulation of hydraulic erosion.

Smoothed Particle Hydrodynamics (SPH) In an SPH system, the state of the system is represented by a number of particles that each store individual physical properties such as mass, density, and velocity. Each particle represents a small volume of the simulated object. Tracking the set of moving particles simulates fluid dynamics. The value of any physical property at a single particle can be calculated by smoothly interpolating the values at the particles in its neighborhood. In the system, time is discretized into small steps. In each time step, the movement of each particle is calculated according to governing conservation laws and the state of the system in the previous time step. SPH is highly robust and can naturally simulate objects with extremely large deformations or composed with various materials.

SPH Applications SPH was initially developed to solve astrophysical and cosmological problems in 3D open space (Gingold 1977, Lucy 1977). SPH has been applied to simulate stellar collisions, supernova, and galaxy collapse (Benz 1988, Hultman 1999, Monaghan 1992). The SPH method has also been applied extensively to in computational fluid and solid mechanics including elastic flow, quasiincompressible fluids, and shock wave simulation (Swegle 1992, Monaghan 1983). Desbrun \& Cani (1999) were the first to use SPH within computer graphics research. Muller et al. (2003) developed interactive methods for simulating and rendering fluids and the interaction between non-elastic solids and fluids (Muller 2004). Solenthaler et al. (2007) proposed an SPH method to model elastic, plastic, and brittle solids and their interaction with fluids. The interaction between multiple SPH fluids with different physical properties was introduced in Muller et al. (2005). Interactive simulations and visualizations of rivers were presented by Hultman \& Pharayn (1999). SPH has also been used to simulate small-scale phenomena, such as porous flows (Lenaerts 2008), bubbles (Hong 2008), and melting and freezing (Wicke 2006).

Hydraulic Erosion Simulation Musgrave et al. (1989) introduced one of the first techniques for simulating erosion of terrains. They represent the terrain as a height fields and model how water dissolves, transports, and re-deposits soil according to the sediment capacity of water and the gradient of the terrain. Since then, several erosion simulation techniques based on height fields or layered height fields have been proposed (Benes \& Forsbach 2001, Neidhold et al. 2005, and Kristof et al. 2009). 
Benes et al. (2006) presented a method that couples fluid simulation and hydraulic erosion on 3D grid cells. Benes (2007) demonstrated a shallow-water model, where water flow between neighboring columns in the height field is calculated by the difference in height values of the columns. Later this method was improved and implemented using the GPU to enhance efficiency (Mei 2007). Wojtan et al. (2007) introduced a method based on cell grids and level sets for simulating various natural phenomena, such as erosion, sediment and acid corrosion. Kristof et al. (2009) presented a simulation method coupling Smoothed Particle Hydrodynamics (SPH) and height fields, using particles to represent fluid and terrain surfaces and height fields to represent terrain volume. However their method is not able to model some natural phenomena including overhangs. Furthermore, their physical model for simulating erosion is not accurate enough for engineering applications.

\section{| REAL-WORLD EROSION EXPERIMENTS}

Our real-world experiments were done in a $0.356 \mathrm{~m} \times 0.61 \mathrm{~m} \times 0.914 \mathrm{~m}$ box (interior dimensions), with a $0.76 \mathrm{~m}$ high and $0.61 \mathrm{~m}$ wide plywood core to partition the space into two distinct zones and serve as a low-permeability core for the levee. The levee was constructed with an $0.203 \mathrm{~m}$ wide crown and has $5 \mathrm{H}: 1 \mathrm{~V}$ slopes (see Fig. 1). The water source was located in the middle of the one end of the box, and after water filled the left half of the box the water ran over the top of the levee and down dry embankment slope scouring the soil. Eventually, a full breaching of the levee occurred, exposing the plywood core. For more details about the physical experiments, please refer to (Gross 2010).

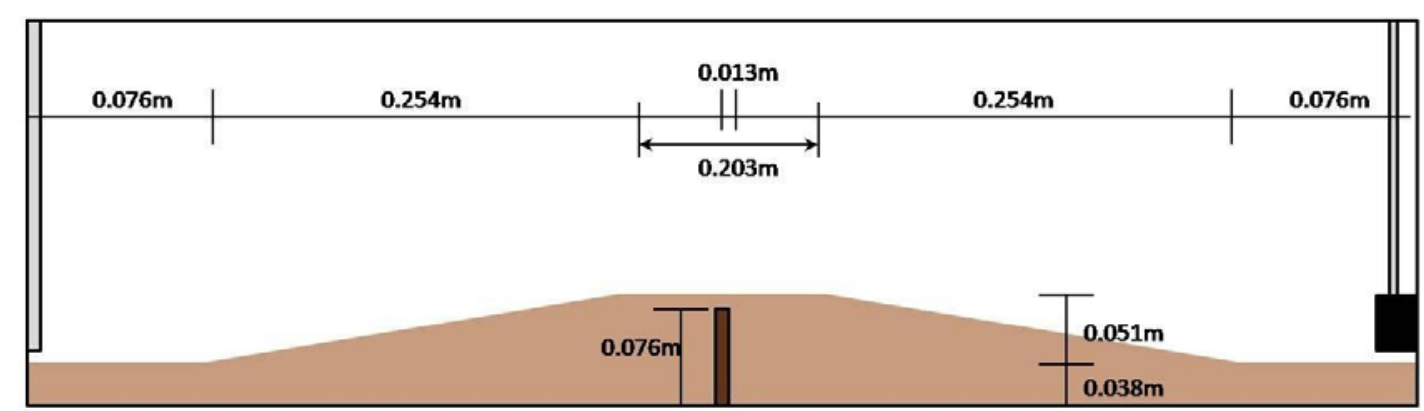

Figure 1. Schematic profile view of physical test setup. The water source is located on the left edge of the diagram, and the sink is on the right edge.

\section{| DATA COLLECTION AND TERRAIN REPRESENTATION}

A 3D laser range scanner was used to collect geometric surface data both during construction of the physical levee model and immediately before and after the experiments. Each scan collects surface data in the form of point cloud. The points are aligned to a regular grid in the XY plane, averaged, and smoothed to fill in holes.

\section{DIGITAL SIMULATION SYSTEM}


In this section we present the details of our digital hydraulic erosion simulation system, including all necessary physical parameters and models.

\section{Fluid Simulation}

Our SPH fluid framework is primarily based on the work of Muller et al. (2003). Fluid behavior is modeled by the Navier-Stokes equation for conservation of momentum:

$$
\rho\left(\frac{\partial v}{\partial t}+v \nabla v\right)=-\nabla p+\rho g+\mu \nabla^{2} v
$$

where $\rho$ is fluid density, $\mathrm{v}$ is velocity, $\mathrm{p}$ is pressure, $\mathrm{g}$ is an external force field and $\mu$ is the dynamic viscosity. In our system, water density is $1000 \mathrm{~kg} / \mathrm{m}^{3}$ and dynamic viscosity is set to be $0.002 \mathrm{~N} \mathrm{~s} / \mathrm{m}^{2}$. Note that mass conservation is automatically preserved in the system according to the nature of SPH; thus, the Navier-Stokes equation that formulates mass conservation can be omitted.

There are three important parameters for any SPH fluid simulation system. The first is particle spacing, which defines the size of the volume represented by a particle and thus ultimately defines the spatial resolution of the simulation. A smaller particle spacing will result in a more accurate simulation; however, as particle spacing decreases, the number of particles increases cubically and thus computational resources (CPU \& memory) will place a lower bound on the particle spacing. We use a particle spacing of $0.004 \mathrm{~m}$, generating approximately 450,000 water particles. Another important parameter is the smoothing length, which defines the neighborhood size of the particles. As in most previous work, we use set the smoothing length to be twice the particle spacing, equal to $0.008 \mathrm{~m}$. The third important parameter is time step. If the time step is too large, the simulation will be inaccurate and an unnecessarily small time step reduces the efficiency of the system. Balancing these two factors, we selected a value of 0.001 seconds for this parameter.

\section{| Implementation of the Source and Sink}

The placement and flow rate of the source and sink can greatly influence the simulation results, therefore it is important to match the parameters of the digital simulation as closely as possible to the conditions of the physical experiment. In the digital simulation, the source is implemented as a number of points in a rectangle where water particles with an initial velocity are generated and added to the simulation at specific time intervals. The initial velocity and the time interval is specified to match the flow rate of the source in the physical experiments. Most of our experiments were conducted with a constant flow rate between $0.010 \mathrm{~L} / \mathrm{sec}$ and $0.015 \mathrm{~L} / \mathrm{sec}$ through a small tube with diameter approximately $0.01 \mathrm{~m}$. To ensure a stable simulation we set the flow rate to match the physical experiments, but used a slightly larger source rectangle for the source $\left(0.0006 \mathrm{~m}^{2}\right)$. To match the pump that removes water from the physical experiment, the sink is implemented as a $1.7^{*} 10^{-6}$ $\mathrm{m}^{3}$ cube, and any water particles that enter this space are deleted from the system.

\section{Erosion Simulation}


In this section, we talk about how erosion simulation is integrated into our fluid system. We first talk about how soil is represented in the simulation and then discuss the physical model we use for simulating erosion.

\section{Soil Representation}

In our system, the terrain is represented as a Segmented Height Fields (SHF), which can represent terrains composed of multiple soil layers and also correctly represents overhangs (Stuetzle et al. 2010). For the purpose of erosion simulation, we convert the SHF into soil particles. As we did for water particles, we need to specify the average spacing between neighboring soil particles. The initial geometry for our simulation is taken from the physical experiment. The resolution of this data is quite high, approximately $0.001 \mathrm{~m}$ between point samples. We were not able to perform the digital simulation at this very high resolution due to the expense of representing and calculating SPH for such a large and dense volume. Thus, we chose to set the soil particle spacing to be $0.003 \mathrm{~m}$, generating about 2,500,000 soil particles. Each soil particle represents a volume of soil and the density of the soil is set to be $1500 \mathrm{~kg} / \mathrm{m}^{3}$.

Unlike the water particles, soil particles never change their position in our simulation. Once a soil particle is fully eroded, we remove it from the simulation. Furthermore, soil particles also serve as boundaries preventing water from penetrating the soil. The repelling force from a soil particle on a water particle is calculated by a penalty-force method (Amada 2006):

$$
\vec{f}=\left(K_{S} d-(\vec{v} \cdot \vec{n}) K_{D}\right) \vec{n},
$$

where $K_{S}$ is the penalty force stiffness, $K_{D}$ is the damping coefficient for the velocity $\mathbf{v}$ of an approaching fluid particle, $\mathrm{d}$ is the penetrated distance measured normal to the boundary, and $\mathbf{n}$ is the unit-length surface normal. In our simulation, $\mathrm{K}_{\mathrm{S}}$ is set to be $1.5 * 10^{5}$ and $\mathrm{K}_{\mathrm{D}}$ is 150 .

\section{| Physical Model}

Based on numerous experiments using various soil samples from New Orleans area, Briaud et al. (Briaud \& Chen 2006, Briaud et al. 2008) determined the relationship between the hydraulic shear stress applied by the water flowing over the soil and the corresponding erosion rate experienced by the soil, namely erodibility. Although no explicit erosion formula is provided in these publications, we use the presented data to estimate this relationship for different soils. Briaud \& Chen defined several different categories of soil based on their erodibility. We estimated the types of the two soils used in our physical experiments with respect to these categories and fit linear erosion functions to the data for those materials (Fig. 2). We denote $\mathrm{z}$ as erosion rate $(\mathrm{mm} / \mathrm{hr})$ and $\tau$ as hydraulic shear stress $(\mathrm{Pa})$. The erosion function for our sand material is:

$$
z(\tau)=187.0 * \tau+0.1
$$

And the erosion function for our sand-clay (85\% sand/15\% clay) mixture is:

$$
z(\tau)=93.0 * \tau+0.1
$$




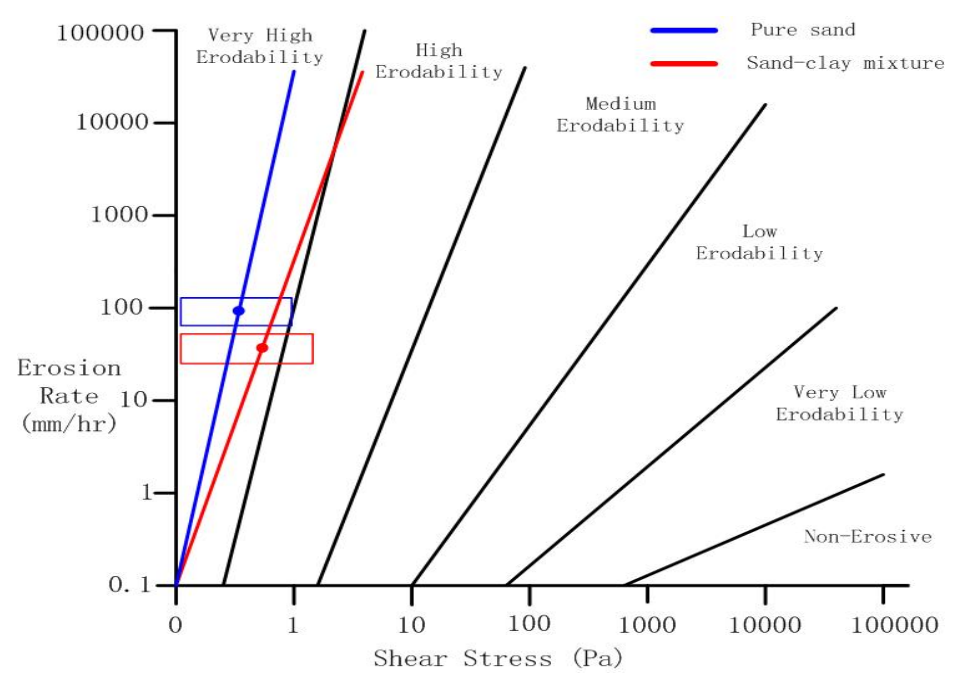

Figure 2. Categories of erodibility and linear erosion functions for materials used in our physical experiments. The boxes of different colors define where the erodibility of the corresponding material lies. The linear erosion function for a single material is calculated by interpolating the origin and a point at the center of the box.

Another important parameter for erosion modeling and simulation is the critical shear stress, which defines the minimum shear stress that can results in erosion. In other words, erosion will only occur for shear stress values equal or greater than $\tau_{c}$. We set $\tau_{c}$ to be 2.0 and 3.0 respectively for the sand and sand-clay mixtures.

Since the erosion functions are functions of shear stress, we need a way to calculate the shear stress applied on a soil particle by a water particle. In our system, shear stress is calculated by:

$$
\tau=K \theta^{m},
$$

where $K$ is a constant set to 1.0 in our simulation, $\theta$ is the shear rate and $m$ is the power-law index, a constant defined by the material of the solid. In our simulation, we treat the soil as pseudo-plastic or shear-thinning fluid, so we assume $m=0.5$. The shear rate $\theta$ is simply approximated by:

$$
\theta=\frac{\left|\overrightarrow{v_{r e l}}\right|}{l},
$$

where $v_{\text {rel }}$ is the velocity of the fluid relative to the solid surface and $l$ is the distance over which the shear is applied.

\section{RESULTS}

Figure 3 presents the results of our computer simulation and physical experiments for a levee model made with pure sand. Similarly, Figure 4 shows our simulation results with a sand-clay mixture. Our computer simulations were run on a computer with four 3.0 GHz CPUs and 8 Gbyte memory. The 10-minute simulation using pure sand took about 192 hours, and the 8-minute simulation with sand-clay 
mixture took about 160 hours. In the 10-minute computer simulation, the number of eroded soil particles is approximately 300,000 (equivalently $0.0081 \mathrm{~m}^{3}$ soil). And in this 8-minute simulation, about 47,000 soil particles (equivalently $0.0013 \mathrm{~m}^{3}$ soil) were eroded.

COMPUTER SIMULATION

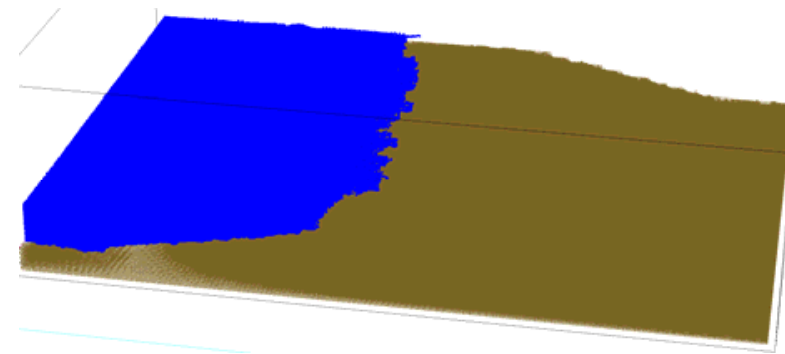

(a)

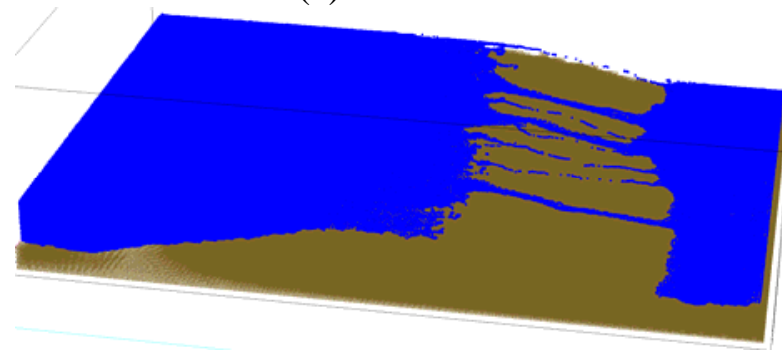

(c)

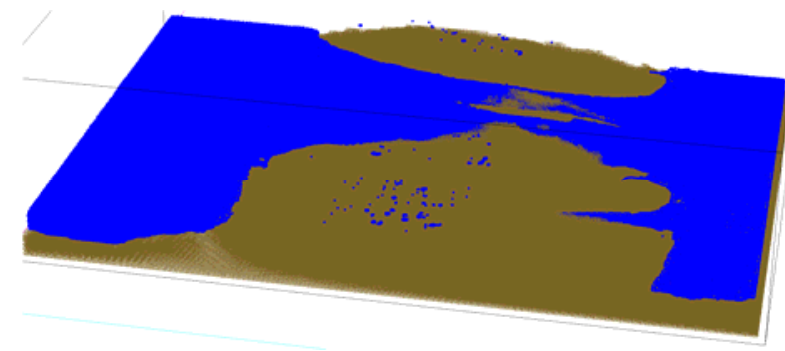

(e)

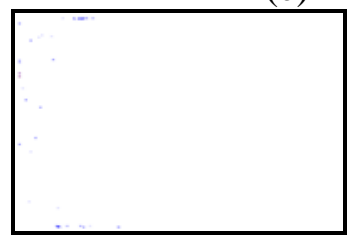

(g)

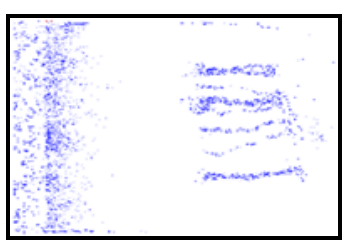

(h)
PHYSICAL EXPERIMENT

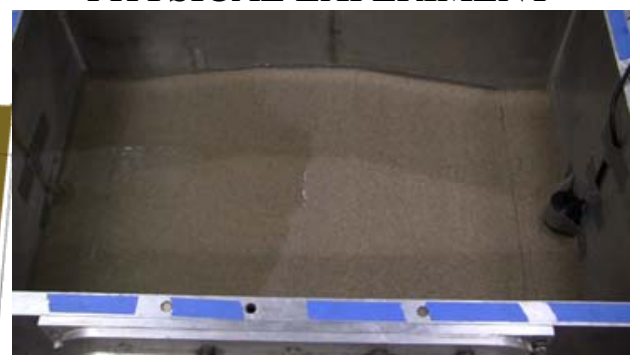

(b)

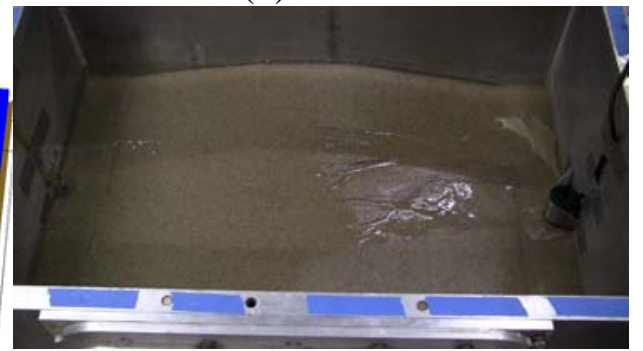

(d)

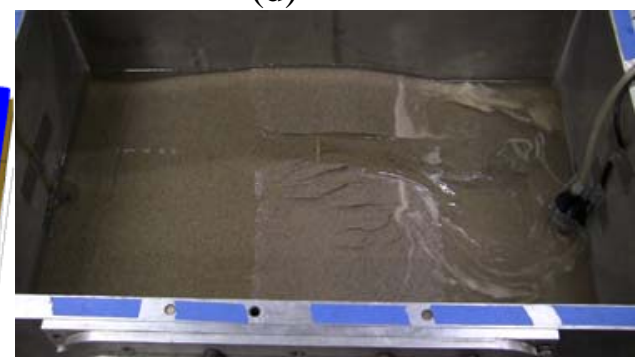

(f)

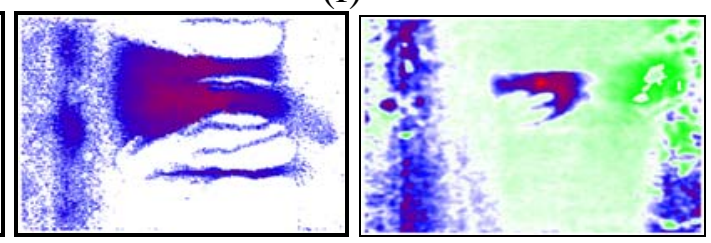

(j)

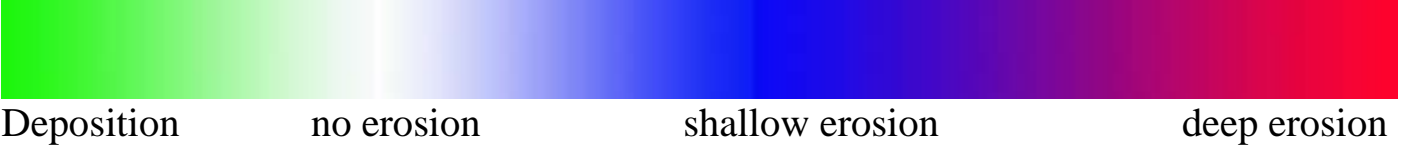

Figure 3. Computer simulation and physical experiment results with pure sand. (a), (c) and (e) are computer simulation results of water and soil represented by particles, while (b), (d) and (f) are physical test results. (a) and (b) were taken at the moment of overtopping. (c) and (d) were taken 1.5 minutes after overtopping. (e) and (f) were taken 10 minutes after overtopping. (g), (h), and (i) visualize the erosion depths corresponding to (a), (c), and (e). (j) shows the depth of erosion 
and deposition in the physical test. White, blue, red and green pixels respectively represent areas with no erosion at all, erosion with depth under 0.010 meters, erosion with depth over 0.010 meters and deposition (see the above color bar). Darker color represents deeper erosion (deposition).

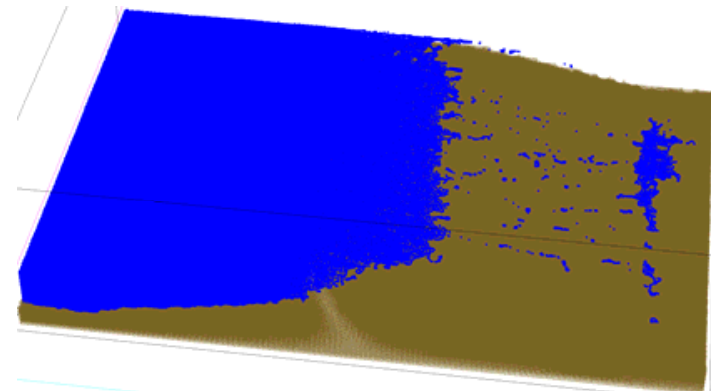

(a)

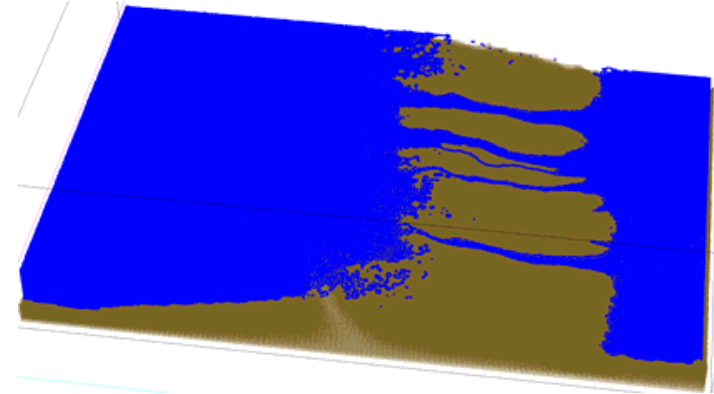

(c)

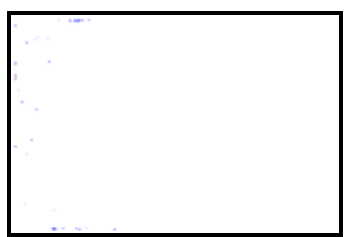

(e)

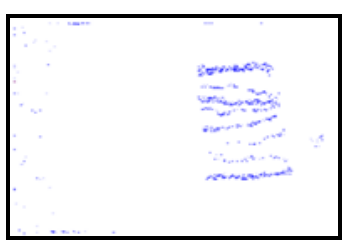

(f)

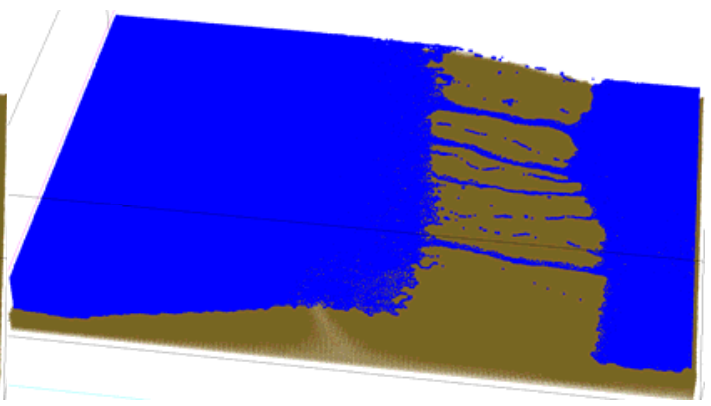

(b)

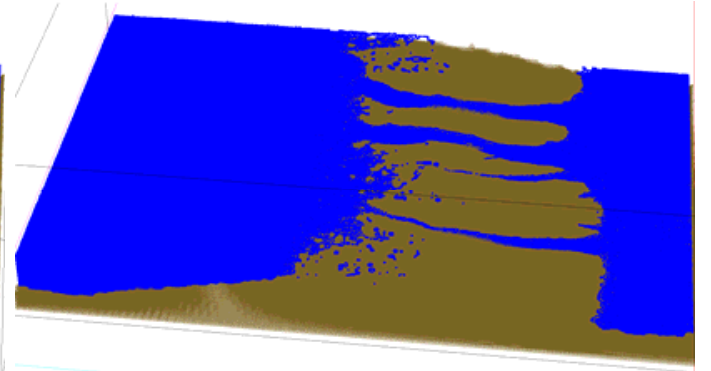

(d)

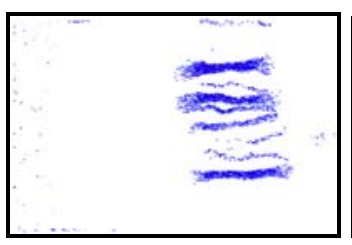

(g)

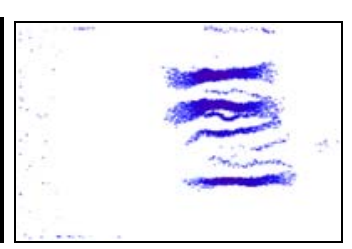

(h)

Figure 4. Computer simulation results with sand-clay mixture. (a)-(d) were taken in particle view respectively 30 seconds, 2 minutes, 5 minutes and 8 minutes after the levee was overtopped. (e)-(f) are images visualizing erosion depths corresponding to (a)-(d).

\section{DISCUSSION}

By comparing the computer simulation results and physical test results, we can see that they look similar to each other. In the physical tests, several narrow shallow rills formed early in the experiment. After a few minutes some of these rills merged to form a single wider and larger gulley and other rills were abandoned and dried up. In our computer simulations, we observed very similar behavior. Furthermore, the shapes of the gullies in our simulated results look similar to those in the physical test. By comparing the simulation results in Figure 3 and 4, we see that the gullies formed in sand are much wider and deeper, which matches the fact that the erodibility of pure sand is larger than the erodibility of sand-clay mixture. Looking at 
the results more closely, we see that the depth of the gullies in the simulation is somewhat larger than in the physical test. There are several possible reasons for this noted difference. We currently do not simulate sediment deposition or soil permeability. Furthermore, estimates of the erodibility of the soils used in our physical experiments may be inaccurate. These are areas for further investigation.

\section{FUTURE WORK}

In future work we will perform physical experiments at high-g in our geotechnical centrifuge, which will scale the earthen structures to the size of actual embankments. Accordingly, we will run digital simulations on large-scale models, and also on small-scale models under high g-level. By comparing the physical experiment and digital simulation results, we will better understand the erosion process and further validate our digital simulation models and implementation.

We are currently working on improving our simulation engine and enabling it to simulate sediment transportation, settling, and soil permeability. We are also enabling it to correctly model and simulate overhangs. Furthermore, we are developing metrics to statistically compare the 3D scans of physical erosion experiments to digital erosion simulations. Finally, using parallel computation, we will improve both the accuracy and efficiency of our digital simulations.

\section{ACKNOWLEDGEMENTS}

This research was supported by NSF grant CMMI-0835762.

\section{REFERENCES}

Amada, T. (2006). "Real-time particle-based fluid simulation with rigid body interaction." In Game Prog. Gems.: 189-205.

Benes, B., and Forsbach, R. (2001). "Layered data representation for visual simulation of terrain erosion." In Proceedings of the $17^{\text {th }}$ Spring conference on Computer graphics.: 80-86.

Benes, B., and Arriaga, X. (2005). "Table mountains by virtual erosion." In Eurographics Workshop on Natural Phenomena.: 33-40.

Benes, B., Tesinsky, V., Hornys, J., and Bhatia, S. K. (2006). "Hydraulic erosion." Computer Animation and Virtual Worlds 17(2):: 99-108.

Benz, W. (1988). "Applications of smoothed particle hydrodynamics (SPH) to astrophysical problems." Computer Physics Communications. 48: 97-105.

Briaud, J. L., and Chen, H. C. (2006). "Levee erosion by overtopping during Hurricane Katrina." Proc., $3^{\text {rd }}$ Int. Conf. on Scour and Erosion, Taylor and Francis, London.

Briaud, J. L., Chen, H. C., Govindasamy, A. V. and Storesund, R. (2008). "Levee erosion by overtopping in New Orleans during the Katrina Hurricane." Journal of Geotechnical and Geoenvironmental Engineering 134.: 618-632.

Desbrun, M., and Cani, M. (1999). "Space-time adaptive simulation of highly deformable objects." Technical Report, INRIA, France. 
Gingold, R. A., and Monaghan, J. J. (1977). "Smoothed particle hydrodynamics: theory and application to no-spherical stars." Mon. Not. R. astr. Soc. 181.: 375-389.

Hong, J., Lee, H., Yoon J., and Kim, C. (2008). "Bubbles alive." In ACM SIGGRAPH 2008.

Hultman, J., and Pharayn, A. (1999). "Realistic and interactive simulation of rivers." Proc. Of Graphics Interface. : 41-48.

Kepfer, P., and Westermann, R. (2006). "Hierarchica, dissipative formation of elliptical galaxies." Astronomy and Astrophysics. 347.: 769-798.

Kristof, P., Benes, B., Krivanek, J., and St'ava, O. (2009). "Hydraulic erosion using smoothed particle hrdrodynamics." Computer Graphics Forum 28(2).: 219228.

Lenaerts, T., Adams, B., and Dutre, P. (2008). "Porous flow in particle-based fluid simulations." In ACM SIGGRAPH 2008.

Lucy, L. B. (1977). "A numerical approach to the testing of the fission hypothesis." Astronomical Journal, 82: 1013-1024.

Mei, X., Decaudin, P., and Hu, B. G. (2007). "Fast hydraulic erosion simulation and visualization on gpu." In Pacific Graphics.: 47-56.

Monaghan, J. J. (1983). "Shock simulation by the particle method SPH." Journal of Computational Physics. 52: 374-389.

Monaghan, J. J. (1992). "Smoothed particle hydrodynamics." Annual Review of Astronomy and Astrophysics. 30: 543-574.

Muller, M., Charypar, D., and Gross, M. (2003). "Particle-based fluid simulation for interactive applications." In Proceedings of the 2003 ACM SIGGRAPH/Eurographics Symposium on Computer Animation.: 154-159.

Muller, M., Schirm, S., and Teschner, B. (2004). "Interaction of fluids with deformable solids." Journal of Visualization and Computer Animation.: 159171.

Muller, M., Solenthaler, B., Keiser, R., and Gross, M. (2005). "Particle-based fluidfluid interaction." In Proceedings of the 2005 ACM SIGGRPH/Eurographics symposium on Computer animation.: 237-244.

Musgrave, F.K., Kolb, C. E., and Mace, R.S. (1989). "The synthesis and rendering of eroded fractal terrains." In Proceedings of the $16^{\text {th }}$ annual conference on Computer graphics and interactive techniques.: 41-50.

Neidhold, B., Wacker, M., and Deussen, O. (2005). "Interactive physically based fluid and erosion simulation." In Eurographics Workshop on Natural Phenomena.: 25-32.

Solenthaler, B., Schlafli, J., and Pajaroa, R. (2007). "A unified particle model for fluid-solid interactions." Computer Animation and Virtual Worlds.: 69-82.

Swegle, J. (1992). "SPH in tension." Memo, Sandia National Laboratories, Albuque, USA.

Wicke, M., Hatt, M., Pauly M. Muller, M., and Gross, M. (2006). "Versatile virtual materials using implicit connectivity." In Proc. Of Eurographics Symposium on Point-Based Graphics .: 137-144.

Wojtan, C., Carlson, M., Mucha, P.J., and Turk, G. (2007). "Animating corrosion and erosion." In Eurographics Workshop on Natural Phenomena.: 21-29. 